\title{
Desafios Colocados à Investigação ao Contexto e ao Investigador: Adaptação ao Investigador em Dificuldades e em Contexto Difícil
}

\begin{abstract}
Serafim Camalhão ${ }^{1}$
${ }^{1}$ Mestre em Sociologia das Organizações do Trabalho e do Emprego, Doutorando em Sociologia ISCTE IUL, Portugal | serafimleopoldo@hotmail.com | https://orcid.org/0000-0002-8521-7553

Resumo: Em 2020 estava previsto realizar um workshop para apresentar uma aplicação prática da metodologia adaptada ao contexto e ao investigador. A pandemia cancelou esta atividade. O principal e único objetivo desta metodologia proposta é tornar a investigação acessível a todos. Nos últimos dois anos foram reunidas todas as contribuições dos oito anos em que foi criada esta abordagem, com o objetivo de criar um projeto de intervenção social que procura ajudar uma aluna com grandes dificuldades para fazer a sua investigação. Deste material geraram-se dois artigos, um correspondente a uma parte teórica, outro de natureza prática. Nesta parte prática partiu-se da investigação ação como forma de intervenção no campo da investigação social. O conceito central desta intervenção é o investigador em contexto difícil. Aqui quer-se distanciar da imagem do que tem acontecido, nesta pandemia, investigadores que não conseguem chegar aos participantes e ir para o terreno devido às regras distanciamento e isolamento social. Muitos métodos e técnicas deixaram de ser utilizadas tal qual foram desenhadas. O que é que há de estranho nisto? Não há investigação sem incerteza, dificuldades e imperfeição. Muitos investigadores estudam campos de difícil acesso, possuem recursos limitados e fazem as suas atividades a título parcial. A pandemia criou as mesmas situações, para muitos investigadores, de um investigador em dificuldades e em contexto difícil. Para este trabalho criou-se Mariana uma aluna virtual, que simula um aluno improvável, mas trabalhadora, organizada e esforçada. Os participantes no evento vão tomando o lugar desta aluna e do seu orientador vão construindo o seu plano de intervenção com as soluções perante um cenário estranho e heterogéneo. No final resulta uma teoria substantiva orientada para a prática e realidade.
\end{abstract}

Palavras-chave: Investigação; Dificuldades; Intervenção; Adaptação; Contexto.

Challenges Putted to Research Adapted to the Context and Researcher: Adaptation to Researcher in Difficulties and in Difficult Context.

\begin{abstract}
In 2020, it was planned to do a workshop, to present a practical application of methodology adapted to context and researcher. The pandemic cancelled these activities. The main and only objective of this proposal is turning the research accessible to all. In the last two years were gathered all the contribution from the eight years that this methodological approach was created, with the objective to construct a social intervention to help student in great difficulties to do her research. From this material it was generated two articles, one more theoretical and the other practical. In this practical part, it was started from action research as a form to intervene in the field of the social research. The main concept of this intervention is the researcher in difficult context. Here it is wanted to distance from the image of it has happened, in this pandemic situation, researchers that can't get closer to the participants and go to the field, due to the distance rules and social isolations. Many methods and techniques ceased to be used in the same way they were drawn. What is strange in this? There isn't research without uncertain, doubt and imperfection. Many researchers study difficult access fields, have limited resources and do research in partial status. The pandemic created the same situations, many researchers, of one researcher in difficulties and in difficult context. To this work it was created Mariana, one virtual student, that simulate an unlikely student, but hard worker, organized and dedicated. The participants of this event, take the place of this student and his director, constructing his intervention plan with the solutions to strange and heterogeneous scenario. The outcome is substantive theory oriented to the practice and reality.
\end{abstract}

Keywords: Research; Difficulties; Intervention; Adaptation; Context.

\section{Introdução}

Desafios colocados à investigação ao contexto e ao investigador: adaptação ao Investigador em dificuldades e em contexto difícil, é o texto de um workshop que foi adiado. $\mathrm{O}$ evento tinha uma base pedagógica e de intervenção com o objetivo de ajudar investigadores em situações e contextos difíceis. 
A pandemia com o Covid 19 afetou a investigação social, na perspetiva deste texto teve o efeito de acordar a consciência que fazer investigação não é uma atividade fácil.

Fazer investigação é uma atividade exigente, necessitando o investigador de um conjunto de competências para construir um projeto de investigação que para o colocar em prática necessita (Deslandes, 2016): escolher um tema, transformá-lo numa problemática; construir um enquadramento teórico, construir hipóteses ou proposições, escolher uma metodologia, métodos e técnicas a aplicar no terreno; como analisar e apresentar os dados. A fase de projecto é puramente académica e principalmente teórica, quando se coloca este execução notam-se as diferenças entre o que se realizou e projectou.

O termo investigador em dificuldades e contextos difíceis é abrangente, inclui o não acesso a financiamento, o campo que se torna difícil, o estudante pouco preparado para fazer investigação, e o trabalhador estudante. O Covid 19 colocou o investigador numa situação em que este não tem acesso ao campo, pelo que não pode fazer investigação. Hammersly \& Atkinson (2019, pp. 44 - 61) falam em acesso ao campo onde acontece, no uma contante negociação e adaptação. Nesta situação o investigador perdeu a proximidade física, o acesso aos locais e o pretenso controle da informação que recolhe. A solução para este problema passa por alargar o conceito de campo no sentido etnográfico inclui todas as técnicas e métodos, assim como fontes de recolha de informação, como por exemplo sites da internet e o facebook (Przybylski, 2021).

Este texto corresponde à segunda parte do workshop, tem um carácter prático orientado a solução de problemas, diferente do primeiro texto, com carácter mais teórico. Ambos os textos são resultado dos trabalhos efectuados sob a metodologia adaptada ao contexto e investigador com os autores utilizados, actualizados e acrescentados. Há uma orientação para a prática, com a introdução de traços da metodologia da investigação acção a outras como a Grounded Theory, a fenomenologia, a etnometodologia e formas mais clássicas de fazer ciência.

Utiliza-se aqui a figura da criação de um cenário (Guerra, 2002, pp. 159 - 162), no qual se cria um modelo de uma situação problemática, com todas as variáveis e contexto associado muito próximo das dificuldades de muitos trabalhadores estudantes ao que se acrescenta a pandemia. É mais uma simulação em que se criou uma situação, à qual se criam várias respostas. Inclui-se a rotina básica da investigação acção, composta por olhar, pensar e agir num acto de inclusão e participação (Stringer, 2014, p. 35). Convidase cada investigador, orientador ou director de investigação e participantes construírem uma investigação simultaneamente una e diversa.

A estrutura deste artigo tem a estrutura prática do workshop compostas por três partes. A primeira parte é a apresentação do cenário, a situação de Mariana trabalhadora estudante, mãe, esposa e mulher de família. Na segunda parte, vai-se apresentar a forma como a investigação-acção vai acontecer nas diversas fases da ajuda a esta aluna. Por último as conclusões que se queriam retirar no final do workshop.

\section{Cenário do Investigador em Dificuldades e em Contexto Difícil}

Na metodologia adaptada ao contexto e ao investigador é usual utilizar situações reais ou baseadas em casos reais que servem de modelo para os artigos. No artigo "Dilemas de Maria" (Camalhão \& Camalhão, 2018) relatava as dificuldades de uma investigadora com Paralisia Cerebral e Baixa visão, no texto (Camalhão \& Camalhão 2019) o "Acesso da investigação para todos" partiu-se do contexto de uma aluna de mestrado que tem que fazer a sua dissertação e junta os papeis de estudante, investigadora, trabalhadora, mãe, esposa, mulher de família.

Esta aluna virtual chamou-se de Mariana, simulando-se um diagnóstico, de forma a desenhar um processo de intervenção que a ajude a percorrer todo processo de investigação e terminá-lo com sucesso. 
Identificam-se as necessidades desta aluna, procuram-se os pontos fortes, fracos, das oportunidades e ameaças que colocam em causa o sucesso da investigação (Guerra, 2002, pp. 129 - 134).

Aos participantes do workshop seriam apresentados quatro quadros para discussão:

1- A dificuldade da aluna em conjugar o estudo com as tarefas de mãe, professora, esposa e mulher de família. É um problema de gestão de tempo, o que consegue resolver na fase académica.

2- A aluna tem que ter a noção e as competências necessárias a fazer investigação, saber o quer fazer, deve fazer e é capaz de fazer (Rallis \& Rossmann, 2017). É uma aluna que esteve muitos anos afastada do meio académico, é a primeira vez que faz a investigação, não é uma investigadora profissional a tempo inteiro. Não teve acesso a uma bolsa de investigação.

3- A aluna não tem uma ideia do que quer fazer, mas certamente implica estudar uma pequena comunidade escolar, que conhece (Robson \& McCartan, 2016) e é capaz de estudar utilizando o seu tempo livre. Metodologicamente quer utilizar entrevistas (Flick, 2018, pp. 216 - 252) presencialmente, mas não sabe o que pode e quer fazer.

4- Quando começa a pensar a sua investigação começa a Pandemia por Covid 19. Colocam-se inúmeros problemas metodológicos. Os campos anteriormente acessíveis tornaram-se parcialmente incessíveis, e implicam a procura de novas soluções, mesmo assim, não há garantias de reunir a informação suficiente.

Existe um problema com dimensões sociais, académicas e de investigação. Aparentemente criou-se uma situação em que não existem condições para realizar qualquer investigação. Na metodologia adaptada ao contexto e ao investigador, não existem condições rígidas, ou prévias para fazer investigação (Camalhão \& Saraiva, 2020). Toda a construção faz-se em sobre as características do investigador e do contexto, logo não existem condições impossíveis.

\subsection{Pré-Projeto, um Caminho Autobiográfico, a Descoberta do Eu}

A primeiro desafio que se colocou à aluna foi decidir o que fazer. A parte académica está a terminar, e ela teve de escolher ou foi-lhe atribuído um orientador. (Camalhão \& Camalhão, 2019) Este pede-lhe para fazer um pré-projeto, procura-se fazer um pequeno diagnóstico, do que aluno quer e sabe fazer. Procede-se num primeiro contacto informal através de um diálogo, para depois formalmente preencher de um formulário.

Utiliza-se a analogia da Sociologia das Organizações, com a figura do diagnóstico (Hinault, Osty \& Servel, 2019) onde é preciso resolver problemas e redinamizar o seu funcionamento em direção a um objetivo específico. É preciso compreender os participantes nos aspetos objetivos e subjetivos para intervir posteriormente numa estrutura participativa. O papel do orientador é intervir positivamente nos seus alunos de forma a conseguir entregar as suas Dissertações ou Teses de Mestrado. O primeiro passo começa por compreender cada aluno orientado.

Neste caso, a aluna, já vem com uma ideia do que quer fazer e onde, mas em termos teóricos e metodológicos está tudo muito superficial e impreciso. Poder-se-ia ter um quadro pior, com um aluno onde não se tinha a noção do queria fazer. No Workshop dividíamos o grupo dos participantes em dois, um pondo-se no lugar do orientador $e$ outro na aluna. Como procederiam? 
Na parte teórica um dos pontos apresentados era o que é ser-se humano. Com base em Habermäs (2020) e Ricoeur (2019) significa que é ser-se finito desejando querer atingir o infinito, e nisto fazer escolhas racionalizando-as. Em ambos os autores não há uma objetividade total já tal leva a outros aspetos emocionais, afirmação pessoal, sentimentais, a procura do prazer, que são subjetivos. Na prática significa que quer no caso da aluna, como qualquer aluno que não sabe o que quer, vai escolher um tema apontando para o infinito onde se vai perder. Segundo Quivy, Market \& VanCampenhoud (2017) a tendência dos alunos, e desta aluna em particular, em querer atingir a perfeição que não existe, leva a nunca acabem os seus trabalhos. A resposta para este problema está em quadrar e delimitar a investigação para que esta seja exequível.

$\mathrm{Na}$ primeira fase da intervenção, deseja-se manter o campo em aberto. Os alunos tomem conhecimento de todas as teorias e metodologias que existem no campo que querem estudar para depois fazerem as suas escolhas. É a revisão da literatura no sentido amplo, porque dão aos alunos competências amplas que os vão ajudar ao longo do processo de investigação. É a revisão da literatura no sentido amplo, porque dão aos alunos competências amplas que os vão ajudar ao longo do processo de investigação. A maior parte desta revisão surge no sentido (Yin, 2016, pp. 71-79) em que as leituras incidem especificamente numa dada área em função de uma pergunta de partida, e problema. Ao aluno é proposto que durante mês leia livremente sobre o tema que quer estudar, veja superficialmente todas as teorias associadas e todas metodologias utilizadas no que gostaria de fazer (Bentz \& Shapiro, 1998) para que mais tarde possa tomar consciência das suas decisões. O orientador diante dos pontos fracos pode intervir dando referências bibliográficas como ajuda.

No workshop apresentam-se as sugestões e resultados do orientador e da aluna que têm de explicar o porquê das suas escolhas. Apresentam-se os percursos de vida (Bertaux, 2016) que influenciaram as suas decisões. Um momento auto etnográfico (Moriceau, 2019) onde se reflete sobre a importância da vida dos investigadores, na forma como se tomam a decisões e reflete-se sobre o que se faz. O investigador é parte da investigação.

Orientador e aluna tomam consciência das suas decisões e intervenções. O orientador convida a aluna a tornar mais claro aquilo que quer, melhorar a pergunta de partida, transformar o pré-projeto num projeto. A aluna quer estudar o tema da inclusão escolar do aluno com Paralisia Cerebral, quer fazer um estudo de casos, e vai estudar uma escola. Justifica a sua escolha porque profissionalmente é professora, tem com alunos esta problemática; é uma ativista dos direitos dos deficientes; na família tem um caso com Paralisia Cerebral onde tem alguma intervenção direta. Academicamente não encontrou muitos estudos que a ajudassem a lidar com estes alunos, precisava ter mais elementos.

\subsection{A Fase de Projeto}

$\mathrm{Na}$ fase projeto é preciso reduzir a investigação a uma dimensão viável. Faz-se a revisão de literatura em sentido estrito. Parece ser uma tarefa burocrática, que seguem os passos dos manuais de metodologia (Mace \& Pétry (2000), sem explorar a sua riqueza, transformando o projeto de investigação num livro de receitas. Fazer um projeto de investigação, é mais que seguir uma receita ou aplicar no campo teorias, métodos e técnicas previamente certificados, com a promessa de sucesso garantido. Moustakas (1994, pp. 68 - 83) utiliza os termos intencionalidade, noema e noesis, significam que o ato de fazer investigação passa pelos sentidos, pelas perceções, sentidos, sensações e pensamentos. Um projeto de investigação na sua construção tem que ter um significado, tendo por base a sua identidade e temporalidade. O significado e a intencionalidade, são comuns com o orientador, aluno e meio académico. 
A aluna com muita leitura, estruturação conseguiu um projeto quase perfeito. Utilizou a condensação, com o recurso à pergunta de partida com uma revisão de literatura efetuada de modo indutivo (Miles, Huberman \& Saldaña, 2020) onde os diversos elementos excluídos continuaram presentes nos elementos mais significativos da investigação. Todos os elementos de um projeto ficaram naturalmente delimitados. Utilizou-se o método comparativo (Ragin, 2014) e comparam-se os recursos da aluna com os recursos exigidos por teorias, métodos e técnicas. A aluna ( Yin, 2016) foi ao campo fazer um pequeno estudo piloto noutra escola, o que levou ainda a outras reduções.

Os participantes do workshop simularam que a aluna iria fazer um estudo de caso (Yin, 2018) centrado numa escola onde estudaria a inclusão escolar de um ou mais alunos com Paralisia Cerebral, e utilizaria (Ghiglione \& Matalon, 1992) entrevistas semiestruturadas. Dadas as limitações do tempo do evento não daria para se aprofundar muito mais, a não ser que teria um universo e amostra simulada, acessível com uma grande flexibilidade de gestão do tempo.

\subsection{O Trabalho de Terreno no Campo da Pandemia}

O trabalho de campo começou em Fevereiro de 2020, sendo que foi Dezembro de 2019 quando a pandemia começou. A aluna começou a fazer as suas entrevistas, a sua amostra tinha 16 participantes identificados. Tinha presente a duração de quatro meses neste trabalho, mas de repente com cinco entrevistas feitas, tornou-se difícil chegar às pessoas, e o distanciamento social obrigatório. Questões novas surgiram, a nível de orientação e de acesso às pessoas.

Neste momento a aluna pensou que o seu trabalho de investigação tinha acabado. $\mathrm{Na}$ prática, aconteceu o que Frankel (2003) descreve como um vaso quebrado em que o psicanalista ajuda o seu cliente a juntar todas as suas peças. Esse papel cabe ao orientador ajudar a aluna que se sente perdida e a esta repensar a sua investigação. A mesma autora (Frankel, 2017) refere que vida a é atravessada por momentos de incerteza, dúvida e escuridão, tudo está sujeito à mudança. A aluna acreditou piamente que se seguisse todas as regras académicas teria o sucesso garantido. Fazer investigação é um processo dinâmico, no qual o investigador tem que se adaptar ao campo estudado. Isto implica negociar o acesso ao campo para conseguir a adesão dos participantes e obter o seu consentimento na participação na investigação (Hammersley \& Atkinson, 2019). Todos os métodos e técnicas necessitam de alguma adaptação aos participantes (Ghiglione \& Matalon, 1992) e as condições do campo. Moustakas \& Moustakas (2004) indica que o ser humano cresce, ao sair da sua zona de conforto que conhece e domina, na investigação é sair da sua zona de conforto, contruir novas relações e lida com areas do conhecimento pouco conhecida.

Perante este cenário os participantes propuseram rever a parte metodológica, utilizando o princípio de Glasser (2001, p. 145) "all is data" não se fechando a qualquer metodologia, ou dado vindo do campo. A situação era heterogenia porque uns aceitaram fazer entrevistas via telefone, outros preferiram fazer um questionário, alguns participaram via chat, outros deram o seu testemunho por escrito, realizaram-se também entrevistas via video-conferência, mas ainda alguns enquanto foi permitido e com as devidas precauções deram entrevistas ao vivo. A resposta a este problema está (Przybylski, 2021) no campo da etnografia no campo onde não existem limites na recolha de informação. Na internet as redes sociais são muitas vezes homogéneas e tão solidas como no campo. A informação da aluna era contextualizada, incluía apenas os participantes que conhecia de contactos prévios. 
Há a consciência se se perde a riqueza presente nas entrevistas clássicas (Ghiglione \& Matalon, 1992), mas mantêm-se os mesmos aspectos, porque fazer uma entrevista é estabelecer uma relação humana e relacionar-se com o outro (Josselson, 2013), Salmon (2016) indica que com as entrevistas online, é possível fazer observações e tirar notas. Gerber, Abrams, Curwood \& Magnifico. (2017) lembram que a finalidade da pesquisa qualitativa é aprender, via informática, não é muito diferente e faz-se com as devidas adaptações.

\subsection{A Analise de Dados}

Os resultados do trabalho de campo apareceram fragmentados (Bauer \& Gaskell, 2002), imagem, som e texto. A imagem destes dados era semelhante (Franquel, 2003) a de um vaso que se parte em mil pedaços, igualmente de várias naturezas. A análise não é uma fase separada, mas sim a integração constante de todos os elementos tal como Glasser \& Strauss (1967) o fazem. Num olhar (Yin, 2016) verifica-se que uma investigação é composta por um conjunto de estudos integrados, bibliográfico/documental, trabalho de campo e análise de dados.

$\mathrm{Na}$ análise voltou-se ao exemplo do vaso quebrado em mil pedaços, como imagem de um objecto do conhecimento muito fragmentado, combinado com modelo de análise (Saldaña (2016) composto por quatro níveis.

O primeiro ciclo de codificação procurou-se saber o que saber e o que se procura. Interroga-se de como é que é o vaso e onde se encontra. O resultado é a construção um instrumento provisório que Schereier (2012) vai progressivamente reduzindo, tornandose mais preciso, ora agindo indutivamente ou dedutivamente. Procede-se como numa escavação arqueológica (Foucault, 2014), estabelece-se o perímetro onde o objecto se encontra, vai-se para o campo apenas com classificações, no caso o conceito de inclusão escolar e paralisia cerebral. $\mathrm{Na}$ medida que se escava surgem os primeiros pedaços, dados que indicam a dispersão e primeira noção real do fenómeno estudado. Suge a fase de transição com o reequacionar da investigação reduzindo a área.

O segundo ciclo de codificação, delimita o campo de investigação (Foucault, 2014), começam a aprofundar escavando no terreno, cada peça ou pedaço tem um significado, contexto histórico, meticulosamente anotado e fotografado, posteriormente juntam-se todos os pedaços. Neste segundo ciclo, a terceira fase, (Clarke, 2015) mapeia-se o terreno, onde todo os elementos teóricos, metodológicos e empíricos são colocados num mapa com as suas relações. A quarta fase é a redacção da dissertação. Todos os elementos dispersos desta investigação apesar de natureza diferente integram-se naturalmente.

\section{Conclusão do Workshop}

No final do workshop os participantes apresentariam o plano de intervenção do orientador utilizado sobre esta a aluna modelo. Algo muito simples, com o formato de uma matriz com cada fase da investigação, o problema apresentado, a intervenção do orientador e a resposta da aluna (Miles, Huberman \& Saldaña, 2020; Stringer, 2014). A Covid 19, foi mais um problema que orientador e aluna tiveram que resolver, no que já era de um investigador em dificuldades, sem bolsa, trabalhador estudante, com família. O cenário já de si catastrófico, fez com que muitos considerassem ser impossível fazer investigação. Nada é impossível quando o ponto de partida é o investigador e contexto que vai ser estudado. Bentz \& Shapiro (1998) apresentam esta mesma ideia, sendo possível misturar-se métodos e técnicas diferentes, mas que é preciso ter consciência das suas decisões. 
O principal desafio que se colocou à aluna, ao orientador, na pele dos participantes do workshop, foi o da unidade da investigação conseguida pela contextualização, onde não (Bentz \& Shapiro, 1998) se exclui nenhuma fonte de informação. Przybylski (2021) por seu lado também refere a possibilidade de combinar várias formas e fontes de informação. Pode parecer que o resultado da investigação e seu desenho parecem confusos, assim como os seus resultados não permitem grandes realizações.

A aluna conseguiu uma teoria substantiva (Glasser \& Strauss, 1967) que representa apenas a inclusão do(s) aluno(s) com Paralisia Cerebral naquela escola. Neste espaço de tempo ganhou competências múltiplas a nível teórico e metodológico. São para esta uma ferramenta profissional. Para a instituição, ficou com elementos para melhorar a inclusão quer de alunos com dificuldades quer alunos com deficiência. Para todos os que contribuíram para a metodologia adaptada ao contexto ao longo dos anos, a defesa que não existem desenhos universais, nem campos perfeitos ou acabados, mas que a investigação constrói-se sobre as características do investigador e do contexto.

\section{Referências}

Bauer, M. W. \& Gaskell, G. (2002). Pesquisa qualitativa com texto, imagem e som: um manual prático (P. A. Guareschi, Trad). Editora Vozes.

Bentz, V. M. \& Shapiro, J. M. (1998). Mindful Inquiry in Social Research. Sage Publications, Ltd.

Bertaux, D. (2016). Le récit de vie (4 ${ }^{\mathrm{eme}} \mathrm{Ed}$.). Malakoff: Armand Colin.

Camalhão, I, Camalhão, S. \& Saraiva, H. (2021). Desafios colocados à investigação e ao investigador: adequação perante a unidade e diversidade (Manuscrpt submited for publication).

Camalhão, S. \& Saraiva, H. (2020). Orientar segundo a metodología orientada para o contexto e o investigador: parte I questões teóricas e metodológicas. New Trends in Qualitative Research, 2, 106-120. https://doi.org/10.36367/ntqr.2.2020.106-120.

Camalhão, I. \& Camalhão, S. (2019). Acesso da investigação para todos: uma metodologia adaptada e dirigida ao ser humano. Educação para o desenvolvimento. $7,54-66$. http://www.joaodedeus.pt/documentacao/revistacientifica/ED_7.pdf.

Camalhão, I. \& Camalhão, S. (2018, Julho, 10 - 12). Dilemas de Maria: manifesto da investigação para todos (Conference session). $7^{\circ}$ Congresso Ibero-Americano em Investigação. Fotaleza. Brasil Qualitativa. https://proceedings.ciaiq.org/index.php/ciaiq2018/article/view/1627/1580

Clarke, A. E. (2015). From grounded theory to situational analysis: whats new? Why? How? In Clarke, A. E., Friese, C., Washburn, R. (eds). Situational analysis in practice: mapping research with grounded theory (pp. $84-118)$. Left Coast inc.

Deslandes, S. F. (2016). O projecto de pesquisa como exercício científico e artesanato intelectual. M. C. S. Minayo (org.) Pesquisa social: teoria método e criatividade. (pp. 29 - 55). Editora Vozes.

Flick, U. (2018). An introduction to qualitative reserch (6 ${ }^{\text {th }}$ Ed.). Sage Publications Ltd.

Foucault, M. (2014). A arqueologia do saber (M. S. Pereira). Edições 70.

Frankel, E. (2017). The wisdom of not knowing: discovering a life of wonder by embracing uncertainy. Shambala Publications.

Frankel, E. (2003). The sacred therapy: Jewish spiritual teachings on emocional healing and inner wholeness. Shambala Publications.

Gerber, H. R., Abrams, S. S. Curwood, J. S. \& Magnifico, A. M. (2017). Conducting qualitative research of learning in on line spaces. Sage Publications, Ltd.

Guerra, I. C. (2002). Fundamentos e processos de uma sociologia de acção: o planeamento em ciências sociais ( $2^{\mathrm{a}}$ Ed.) Princípia.

Ghiglione, R. \& Matalon B. (1992). O inquérito: teoria e prática. Celta Editora. 
Glasser, B. G. (2001). The Grounded Theory Perspective: Conceptualization Contrasted with Description. Sociology Press.

Glasser, B. G \& Strauss, A. L. (1967). The discovery of grounded theory: strategies for qualitative Research. Aldine.

Habermas, J. (2020). Obras escolhidas de Jürgend Habermas volume V: crítica da razão (L. Nahodir Trad.). Edições 70.

Hammersley, M. \& Atkinson, P. (2019). Ethnograthy: principles in practice (4 ${ }^{\text {th }}$ Ed.). Routlege.

Hinaud, A.-C., Osty, F. \& Servel L. (2019). Résister à la comande co-construire la demande. In Hinaud, A.-C., Osty, F. \& Servel L. (Orgs.). Enquêter dans les organizarions: comprendre pour agir (pp. 13 - 19). Presses Universataires de Rennes.

Josselson, R. (2013). Interviewing for qualitative inquiry: a relational approach. Guilford Press

Mace, G. \& Pétry, F. (2000). Guide d'élaboration d'un project de recherche en sciences sociales. de boeck

Moriceau, J.-L. (2019). Explorer sa prope expérience l'autoetnographie: conter soi-même comme un outre. In J.-L. Moriceau \& R. Soparnot (Cood.). Recherche qualitative en sciences sociales: s'exposer, cheminer, réflexir ou l'art de composer sá méthode (pp. 53 - 66). editions ems.

Miles, M. B., Huberman, A. M. \& Saldaña (2020). Qualitative data analysis: a methods sourcebook (4th Ed.). SAGE Publications Itd.

Moustakas, C. \& Moustakas, K. (2004). Loneliness, creativity \& love: awakening meaning in life. Xlibris.

Moustakas, C., (1994). Phenomenological research methods. SAGE Publications Itd.

Quivy, R., Market J. \& Van-Campenhoud, L. (2017). Manuel de Recherche en sciences Sociales. (5 $5^{\mathrm{a}}$ Ed. entièrement revue et augmentée). Dunod.

Przybylski, L. (2021). Hybrid etnography: online, offline, and in the between. Sage Publications Ltd.

Rallis, S. F. \& Rossmann, G. B. (2017). An introduction to qualitative research: learning in the field. Sage Publications, Ltd.

Ragin, C. C. (2014). The comparative method: moving beyond qualitative and quantitative strategies. University of California Press

Ricoeur, P. (2019). O homem falível (H. Barros \& G. Marcelo Trads). Edições 70.

Robson, C. \& McCartan, K. (2016). Real world research: a resource for users of social research methods in applied settins ( $4^{\text {th }}$ Ed.). John Wiley \& Sons Ltd.

Saldaña, J. (2016). The coding manual for qualitative researchers (3 $3^{\text {rd }}$ Ed.). Sage Publications Ltd.

Salmons, J. (2016). Qualitative ressearch on line. Sage Publications Ltd.

Schreier, M. (2012). Qualitative Content Analysis in Practice. Sage Publications Ltd.

Stringer, E. T.(2014). Action Research (4 ${ }^{\text {th }}$ Ed.). Sage Publications Ltd.

Yin, R. K. (2018). Case study research ans applications: design and methods (6 ${ }^{\text {nd }}$ Ed.). Sage Publications Ltd.

Yin, R. K. (2016). Qualitative research from start to finish (2nd Ed.). The Guilford Press. 